\title{
Monitoring the black hole binary GRS 1758-258 with INTEGRAL and RXTE
}

\author{
Katja Pottschmidt ${ }^{* 1,2}$, Masha Chernyakova ${ }^{3}$, Piotr Lubiński ${ }^{4,5}$, Simone Migliari ${ }^{6,7}$, \\ David M. Smith ${ }^{8}$, Andrzej A. Zdziarski ${ }^{5}$, John A. Tomsick ${ }^{9}$, Ingo Kreykenbohm ${ }^{10,11}$, \\ Peter Kretschmar ${ }^{6}$, and Emrah Kalemci ${ }^{12}$ \\ ${ }^{1}$ CRESST and NASA Goddard Space Flight Center, Astrophysics Science Division, Code 661, \\ Greenbelt, MD 20771, USA \\ ${ }^{2}$ Center for Space Science and Technology, University of Maryland, Baltimore County, 1000 \\ Hilltop Circle, Baltimore, MD 21250, USA \\ ${ }^{3}$ Dublin Institute for Advanced Studies, Fitzwilliam Place 31, Dublin 2, Ireland \\ ${ }^{4}$ ISDC Centre for Astrophysics, Chemin d'Écogia 16, 1290 Versoix, Switzerland \\ ${ }^{5}$ Centrum Astronomiczne im. M. Kopernika, Bartycka 18, 00-716 Warszawa, Poland \\ ${ }^{6}$ European Space Agency, European Space Astronomy Centre, Villafranca del Castillo, P.O. Box \\ 78, 28691 Villanueva de la Cañada, Madrid, Spain \\ ${ }^{7}$ Center for Astrophysics and Space Sciences, University of California San Diego, 9500 Gilman \\ Drive, La Jolla, CA 92093-0424, USA \\ ${ }^{8}$ Department of Physics, University of California Santa Cruz, 1156 High Street, Santa Cruz, CA \\ 95064, USA \\ ${ }^{9}$ Space Sciences Laboratory, University of California Berkeley, 7 Gauss Way, Berkeley, CA \\ 94720-7450, USA \\ ${ }^{10}$ Dr. Karl Remeis-Sternwarte Bamberg, Sternwartstr. 7, Bamberg, 96049 Germany \\ ${ }^{11}$ Erlangen Centre for Astroparticle Physics, Erwin-Rommel-Str. 1, 91058 Erlangen, Germany \\ ${ }^{12}$ Sabanci University, Orhanli-Tuzla, Istanbul, 34956, Turkey \\ E-mail: katja@milkyway.gsfc.gov
}

The microquasar GRS $1758-258$ is one of only three persistent black hole binaries that spend most of their time in the hard spectral state. It therefore provides the rare opportunity for an extensive long term study of this state. INTEGRAL has been monitoring the source since 2003 spring during two $\sim 3$ months long Galactic Center viewing epochs each year, currently amounting to 11 epochs, including 2008 spring. Quasi-simultaneous RXTE observations are available as well. We present an analysis of the epoch averaged broad band spectra. Results include the observation of clearly anti-correlated variations of the $3-300 \mathrm{keV}$ flux and spectral softness, and of the occurrence a faint soft state, i.e., an extreme example of hysteretic behavior. The hard source spectrum and long exposures allow us to extend the analysis of the average hard state spectrum to $\sim 800 \mathrm{keV}$, resulting in the possible detection of a non-thermal Comptonization component. We also discuss the peculiar hardness-intensity diagram of this source.

7th INTEGRAL Workshop: An INTEGRAL View of Compact Objects

September 8-112008

Copenhagen, Denmark

*Speaker. 


\section{Contents}

1. Introduction 2

2. Long term lightcurves 3

3. Broad band spectra $\quad 4$

3.1 Epoch averaged spectra 4

3.2 Average hard state spectrum 5

$\begin{array}{lll}\text { 4. A special link in the evolution through the states } & 7\end{array}$

5. Conclusions 9

5.1 Summary 9

$\begin{array}{llr}5.2 \text { Outlook } & 10\end{array}$

6. Acknowledgments 10

\section{Introduction}

Black hole X-ray binaries can display two different basic "states" with respect to their X-ray and radio emission properties. Especially transient sources in outburst show transitional phases between these states that can be weeks long. One of the states is the hard state, where the broad band X-ray spectrum is dominated by emission above $10 \mathrm{keV}$, strong X-ray variability on time scales faster than $1 \mathrm{~s}$ is observed, and steady radio emission is detected. The other one is the soft state, which is dominated in the X-rays by emission below $10 \mathrm{keV}$, does show much less short term $\mathrm{X}$-ray variability, and generally no detectable radio emission. The phenomenology of these states and the typical progression of a transient outburst through the states is well estabilshed, thanks to monitoring observations of many outbursts by RXTE, INTEGRAL, and other missions, see section 4 for more details. However, physical models for the states and transitions are still under development $[20,8]$. Further insight can be expected from studying a source like GRS 1758-258, which is persistent, thus allowing us to study the parameter space of states on time scales of years, and atypical (see, e.g., the "faint soft state" described below), thus placing further constraints on physical models.

GRS 1758-258 was discovered in 1990 during observations of the Galactic Center region performed with the Granat satellite [7, 17]. It is located only $0.66^{\circ}$ from the bright low mass X-ray binary (LMXB) GX 5+1. Its average spectral and temporal properties - an exponentially cutoff power law spectrum $\left(\Gamma=1.4-1.9, E_{\text {fold }}>100 \mathrm{keV}\right)$ and strong short term variability on frequencies up to $10 \mathrm{~Hz}$, respectively - are rather similar to those of Cygnus X-1 [6, 4]. Based on these Xray properties and on the detection of a double-lobed radio counterpart [11], GRS 1758-258 is 


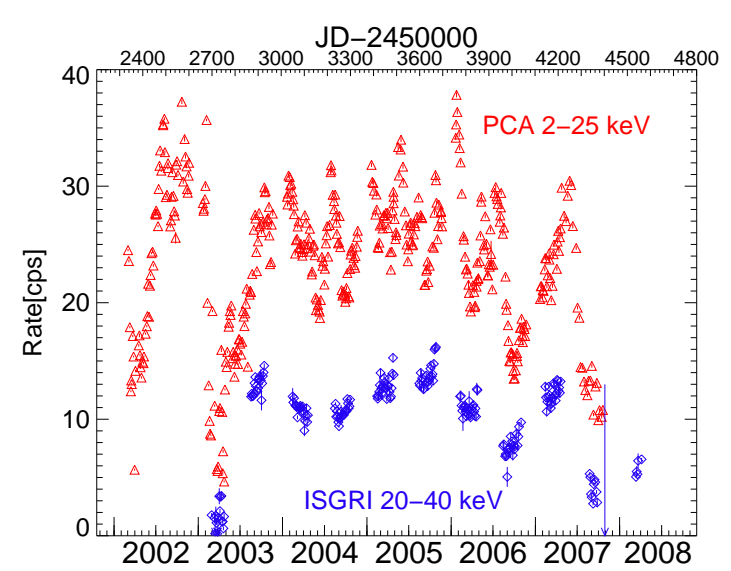

Figure 1: $R X T E-P C A$ (observation-averaged) and INTEGRAL-ISGRI ( $3 \mathrm{~d}$ binning) monitoring light curves of GRS $1758-258$.

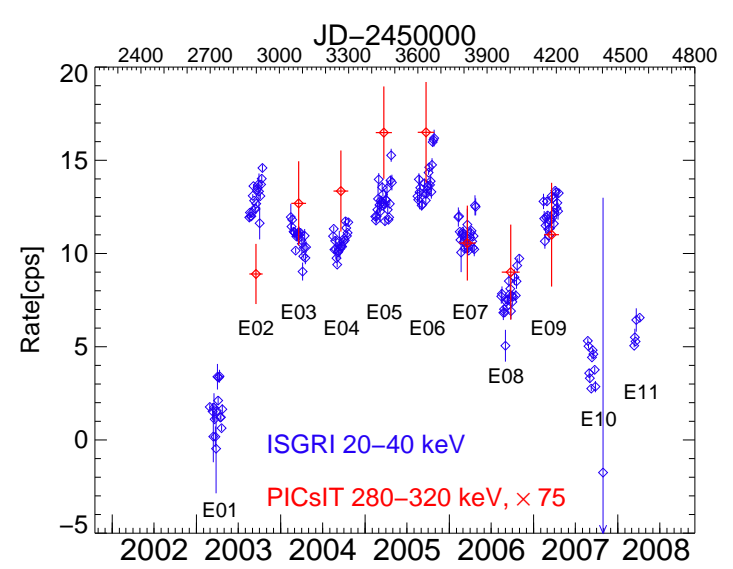

Figure 2: Close-up on the INTEGRAL-ISGRI light curve, including INTEGRAL-PICsIT detections and epoch definitions (E01-E11).

considered to be a microquasar. It is one of only a handful persistent black hole binaries, with Cyg X-1 and 1E 1740.7-2942 being the only other ones predominantly in the hard state, making GRS 1758-258 a prime candidate for studying this complex accretion state. In contrast to the high mass X-ray binary (HMXB) Cyg X-1, however, GRS 1758-258 most likely has a low mass companion [12] and might thus belong to a source class that is usually transient due to Roche lobe overflow accretion.

\section{Long term lightcurves}

GRS 1758-258 has been monitored by RXTE from 1996 to 2007 with about two observations per week and by INTEGRAL from 2003 to today during two Galactic Center viewing epochs per year, each $\sim 3$ months long (Fig. 1, Fig. 2). Already the early RXTE monitoring revealed properties different from those of the canonical black hole hard state: in GRS 1758-258 the observed spectral hardness is correlated with the flux derivative on time scales of days (in the sense that the source becomes softer with declining flux), while Cyg X-1 shows no such hysteretic behavior [15]. The $R X T E$ observations of 2001 also led to the discovery of the phenomenon of the rare faint soft state $[13,16]$. Another occurrence of a faint soft state in 2003 spring is apparent in the light curves (Fig. 1, Fig. 2). Our earlier INTEGRAL-RXTE spectral fits already showed that, like the 2001 faint soft state, this faint soft state shows a reduced bolometric luminosity compared to the hard state, in this case by up to 20\% [9], even though the broad band fit indicates the presence of enhanced black body radiation compared to the hard state, peaking below $1 \mathrm{keV}$. While this is again different from persistent HMXBs, where the soft state is associated with higher bolometric luminosities, it is well within the range of hysteretic behavior displayed by LMXB transients (see, e.g., GX 339-4 [19]). In the following we report on updated results from modeling the epoch averaged broad band spectra as well as on using the hardest epochs to study the spectrum above $300 \mathrm{keV}$. 


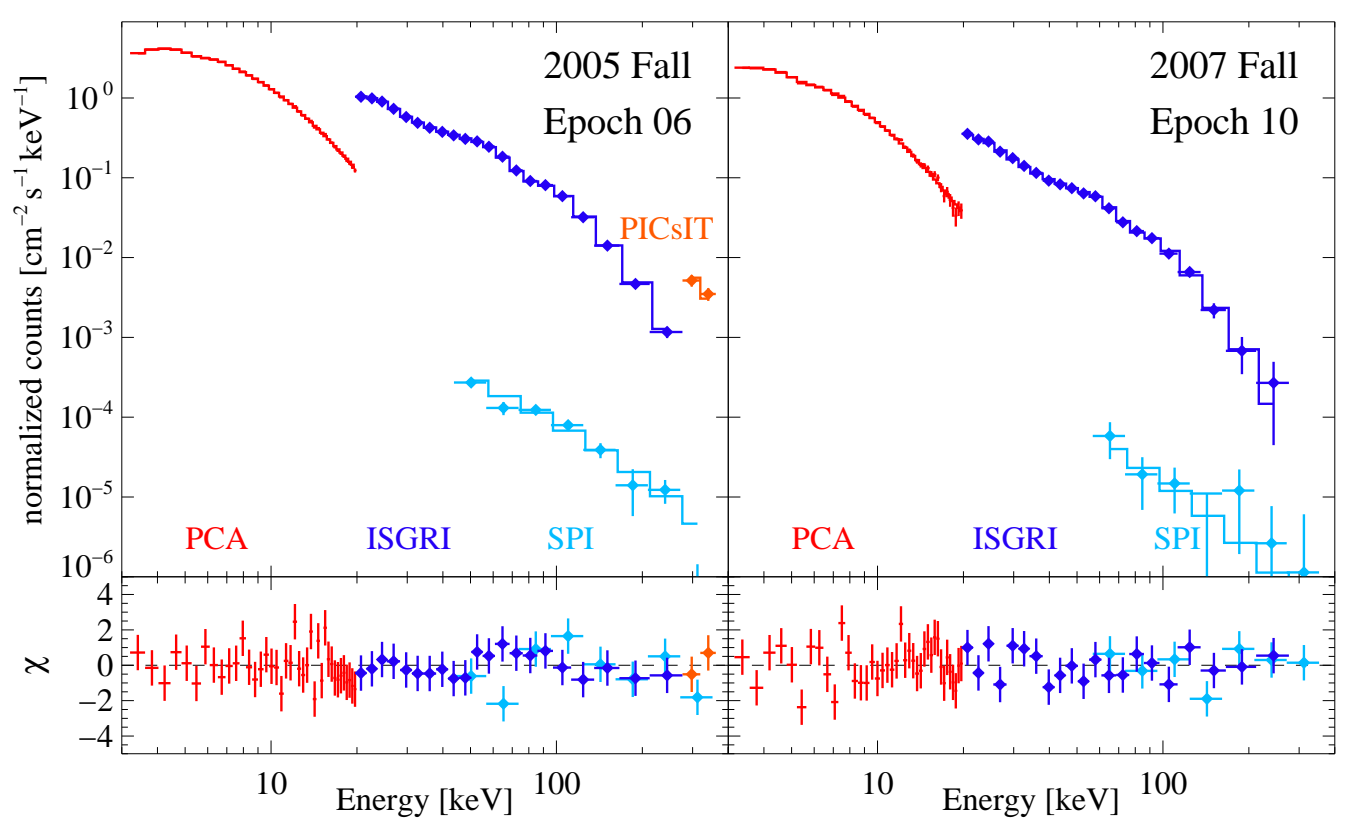

Figure 3: Counts spectra and best fit models (upper panels) and best fit residuals (lower panels) for the quasi-simultaneous RXTE and INTEGRAL data of epoch 06 (left) and epoch 10 (right). The model in xspec notation is constant $\times$ phabs (diskbb+gaussian+power $\times$ highecut).

\section{Broad band spectra}

\subsection{Epoch averaged spectra}

We have modeled the PCA and ISGRI/SPI/PICsIT broad band spectra averaged over each epoch $^{1}$, successfully using either a cutoff power law or thermal Comptonization, plus a disk black body component when required, as well as a weak neutral iron $\mathrm{K} \alpha$ line. See [9] for an earlier version of similar broad band fits for the first four epochs. The PCA spectra are consistent with a column density given by interstellar absorption in the direction of GRS $1758-258$, i.e., $N_{\mathrm{H}}=$ $1.5 \times 10^{22} \mathrm{~cm}^{-2}$. Typical exposures are $\sim 30 \mathrm{ks}$ for the PCA and $\sim 1-2 \mathrm{Ms}$ for the INTEGRAL instruments (due to its smaller FOV JEM-X sees much less exposure from the source than the other INTEGRAL instruments and its data are not included here). Currently the fits for epochs 10 and epoch 11 still contain less data than those typical values since only data from the Galactic Centre Key Programme are included, more exposure will be available after the proprietary period. Note that epoch 10 was the last epoch covered by RXTE monitoring.

Fig. 3 shows the counts spectrum, best fit model, and residuals for two epochs, epoch 06, which shows a comparatively hard spectrum, and epoch 10, which is the second softest epoch after the faint soft state of 2003 (epoch 01). During these two softer epochs $(01,10)$ the source is not detected by PICsIT (for epoch 11 we do not have enough data yet). For the other epochs the most significant PICsIT detections, i.e., measurements in the energy band around $\sim 300 \mathrm{keV}$, are included in the fits.

\footnotetext{
${ }^{1}$ ISGRI and SPI products were obtained with OSA 7.0, for PICsIT see [5], for the PCA see [6].
} 


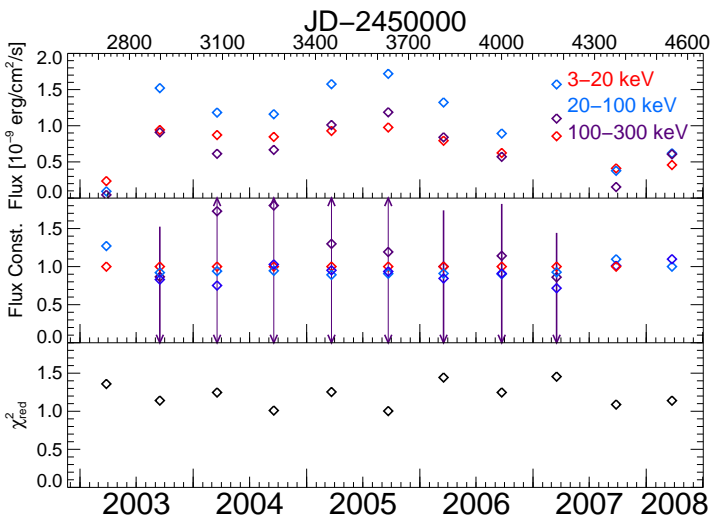

Figure 4: Results of the cutoff power law fits for all epochs. Top: Fluxes (absorbed) in three energy bands. Middle: Flux constants for the PCA (red, set to 1), ISGRI (light blue, set to 1 in 2008), SPI (dark blue), and PICsIT (purple). Bottom: $\chi_{\text {red }}^{2}$ values.

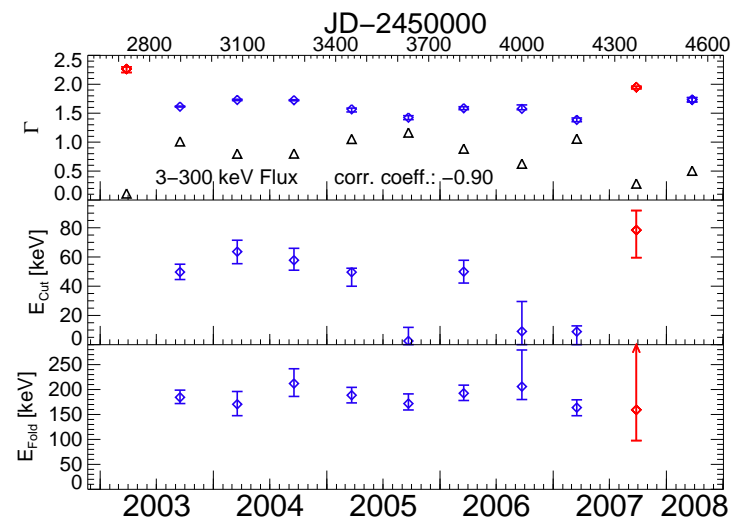

Figure 5: Results of the cutoff power law fits for all epochs. Top: Photon indices $\Gamma$ (blue and red, the red cases indicate softer spectra) and 3-300 keV fluxes [arbitrary units]. Middle: Cutoff energies $E_{\mathrm{Cut}}$. Bottom: Folding energies $E_{\text {Fold }}$.

Fig. 4 and Fig. 5 show results from the cutoff power law fits for all epochs. From Fig. 4 we see that the fluxes in several energy bands up to $300 \mathrm{keV}$ roughly follow the variations seen in the count rate light curves (Fig. 1 and Fig. 2), that the cross calibration of the instruments generally agrees to within $10 \%$ (with the exception of the PICsIT points which cannot be expected to constrain the normalization), and that $\chi_{\text {red }}^{2}$ is $<1.5$ (including $0.5 \%$ and $2 \%$ systematic uncertainties for the PCA and ISGRI, respectively). The upper panel of Fig. 5 shows that, on time scales of months, $\Gamma$ and the 3-300 keV flux are anti-correlated with a correlation coefficient of -0.9 . The middle panel and the lower panel show typical black hole hard state values for the cutoff and folding energies for epoch 02 to epoch 10. No cutoff can be detected in epoch 01 and epoch 11, where the latter is probably caused by the absence of PCA data and the low INTEGRAL exposure available so far.

Results from thermal Comptonization fits for the first four epochs can be found in [9]. Preliminary results for the remaining epochs show similar fit statistics and Comptonization parameters, i.e., describing a hot plasma with optical depths $\tau \sim 0.5-1.5$ and temperatures $k T \sim 40-100 \mathrm{keV}$ for $\chi_{\text {red }}^{2}<1.5$.

\subsection{Average hard state spectrum}

In the next step we averaged all PCA, ISGRI, and PICsIT spectra from epochs during which the source was clearly in the hard state, i.e., from epoch 02 to epoch 09. Currently PCA data sets are included starting at epoch 03 since a different PCU selection has been used for earlier data. (All PCA spectra from epoch 03 onward include only PCU 2 data, i.e., data from the best calibrated PCU. We are in the process of re-extracting the early epochs with this selection as well.) In addition the averaged SPI spectrum is still under investigation and is not yet included here. Caveats and ongoing work aside, we obtain a broad band spectrum with the most sensitive extension to energies $>300 \mathrm{keV}$ to date, thanks to a significant source detection with PICsIT up to $\sim 800 \mathrm{keV}$ (for a detailed report on PICsIT observations, including those of GRS 1758-258, see [5]). 


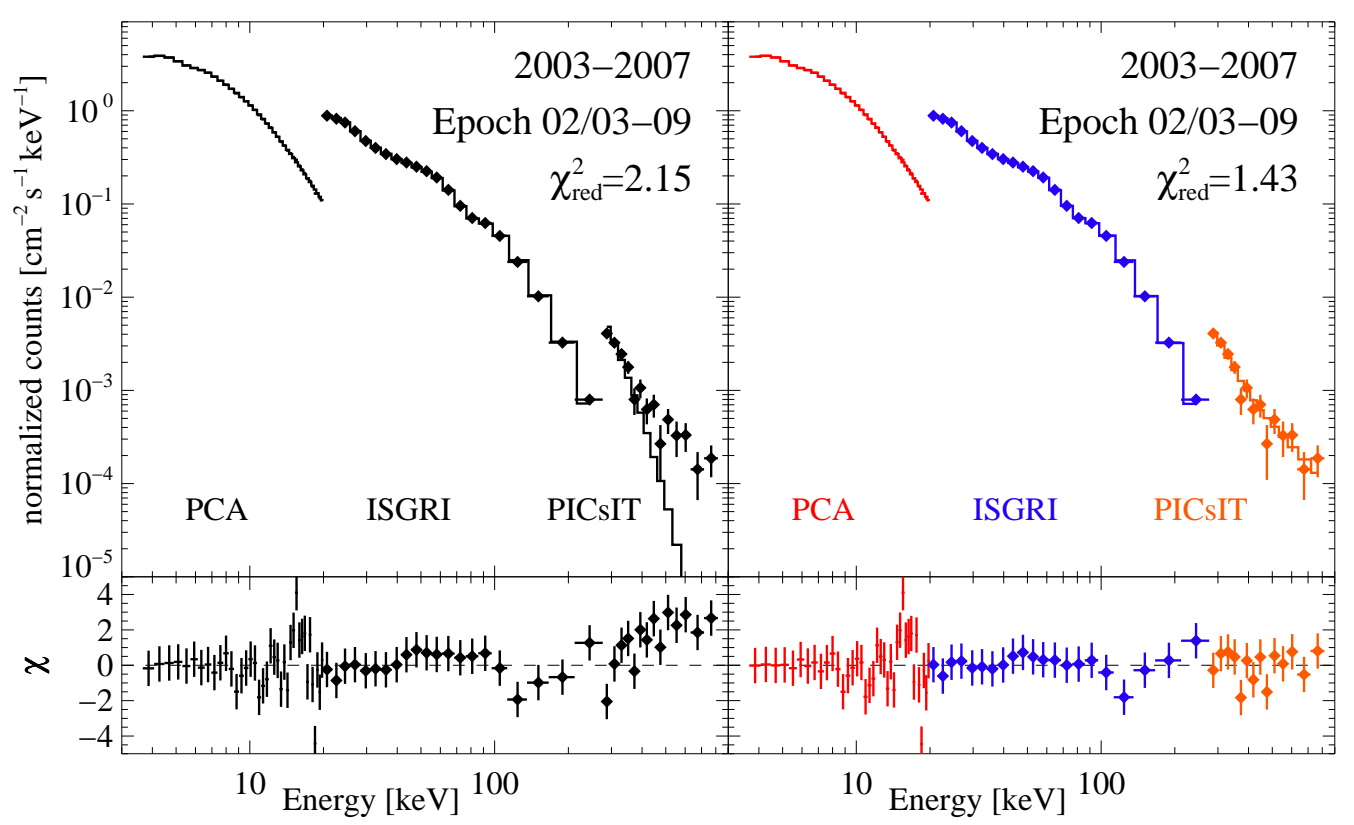

Figure 6: Long term average hard state spectrum of GRS 1758-258, obtained by averaging quasisimultaneous RXTE-PCA and INTEGRAL-ISGRI/PICsIT data from the 2003-2007 epochs. Counts spectra and best fit models (upper panels) and best fit residuals (lower panels) are shown for a thermal Comptonization fit without (left) and with (right) an additional power law component, i.e., in xspec notation the latter model is given by constant $\times$ phabs (diskbb+gaussian+compTT+power).

Applying the two approaches used for modeling the individual epoch averaged spectra, the phenomenological cutoff power law and thermal Comptonization, to this long term average of the hard state spectrum, in both cases clearly cannot explain the data above $\sim 300 \mathrm{keV}$. For the thermal Comptonization model we obtain $\chi_{\text {red }}^{2}=2.15$ (Fig. 6, left) ${ }^{2}$. Another power law component was added to the models in order to describe these residuals. Interestingly this produced qualitatively different results for the cutoff power law and the thermal Comptonization model.

In the latter case the best fit is a stable solution that reproduces the basic plasma parameters from the individual epoch fits $-\tau=1.4 \pm 0.1$ and $k T=41_{-3}^{+5} \mathrm{keV}$ for $\chi_{\text {red }}^{2}=1.43-$, adding a $\Gamma=1.4_{-0.4}^{+0.2}$ power law that dominates the spectrum above $300 \mathrm{keV}$ (Fig. 6 , right). The upper limit for the presence of reflection in the spectrum amounts to $\Omega / 2 \pi<20 \%$. A weak $\sim 0.6 \mathrm{keV}$ black body component is included in the model. This is a classical "hard tail" fit, indicating the possible presence of a non-thermal electron plasma, e.g., in the jet. We caution, however, that this is a preliminary result, there are calibration effects that still have to be evaluated, e.g., the question of the evolution of the ISGRI response with time. In addition the situation is less clear for the cutoff power law case:

Here adding a power law leads to a local $\chi_{\text {red }}^{2}$ minimum of 1.64 , however, for a power law strength marginally consistent with zero and an unusual $\Gamma$ of $-0.2_{-0.3}^{+1.1}$. On the other hand, a fit can be obtained that is formally as good as the thermal Comptonization plus weak power law fit, where the additional power law is strong, with an only roughly constrained $\Gamma$ of $2.0_{-1.4}^{+1.3}$. In this

\footnotetext{
${ }^{2}$ Systematic uncertainties for the PCA were increased to $1 \%$ in the long term average fits.
} 


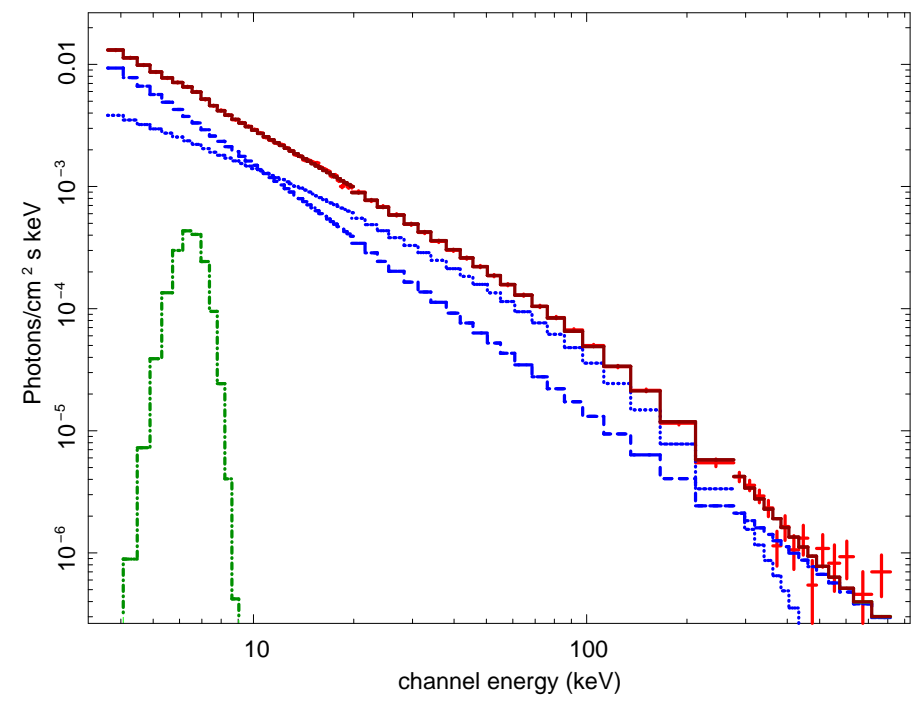

Figure 7: Unfolded data and best fit model applying a cutoff power law plus an additional power law to the average hard state spectrum. The additional power law dominates below $10 \mathrm{keV}$ and above $300 \mathrm{keV}$.

alternative fit the additional power law dominates below $10 \mathrm{keV}$ and above $300 \mathrm{keV}$. The situation is illustrated in Fig. 7. No blackbody component is required in this case. Although the cutoff power law fit is purely empirical, this result points at two different possibilities for the composition of the hot plasma. In order to further investigate this question we will apply models for hybrid thermal/non-thermal plasmas, e.g., eqpair, in future analyses of the average hard state spectrum.

\section{A special link in the evolution through the states}

Outbursts of black hole transients are often characterized by their evolution in the hardnessintensity diagram (HID), typically concentrating on the $\sim 2-20 \mathrm{keV}$ energy range. A few years ago it was realized that outbursts principally follow similar tracks, with a hard rising phase dominated by emission from the corona and/or jet - and a soft declining phase, dominated by emission from the accretion disk - i.e., displaying considerable hysteresis, and with transitional phases at the end of the rise and the decline, where the spectral hardness changes strongly while the X-ray intensity is comparatively stable. Overall the HID is thus roughly "q-shaped" $[2,10]$. In addition the different phases of radio jet emission over the outburst have also been characterized in relation to the HID. This especially includes the existence of the "jet-line", a region in the upper transitional HID branch, where discrete ejections of jet material are most likely, and on the soft side of which the jet is quenched (see [18] for a recent example of the significance of the jet-line in Cyg X-1). Fig. 8 summarizes our current best knowledge of observational properties associated with the evolution of an outburst through the different states and the HID.

Persistent black hole binaries can be expected to occupy a comparatively small area of the " $q$ ". For Cyg X-1 monitoring with RXTE has, e.g., confirmed that the source spends most of its time on the hard state branch above $1-2 \% L_{\text {Edd }}$ with occasional excursions onto the upper transitional branch [18], never clearly descending into the soft thermal branch ("failed state transition", "bright soft state"). Fig. 9 shows the HID of GRS 1758-258 for the RXTE-PCA monitoring during 


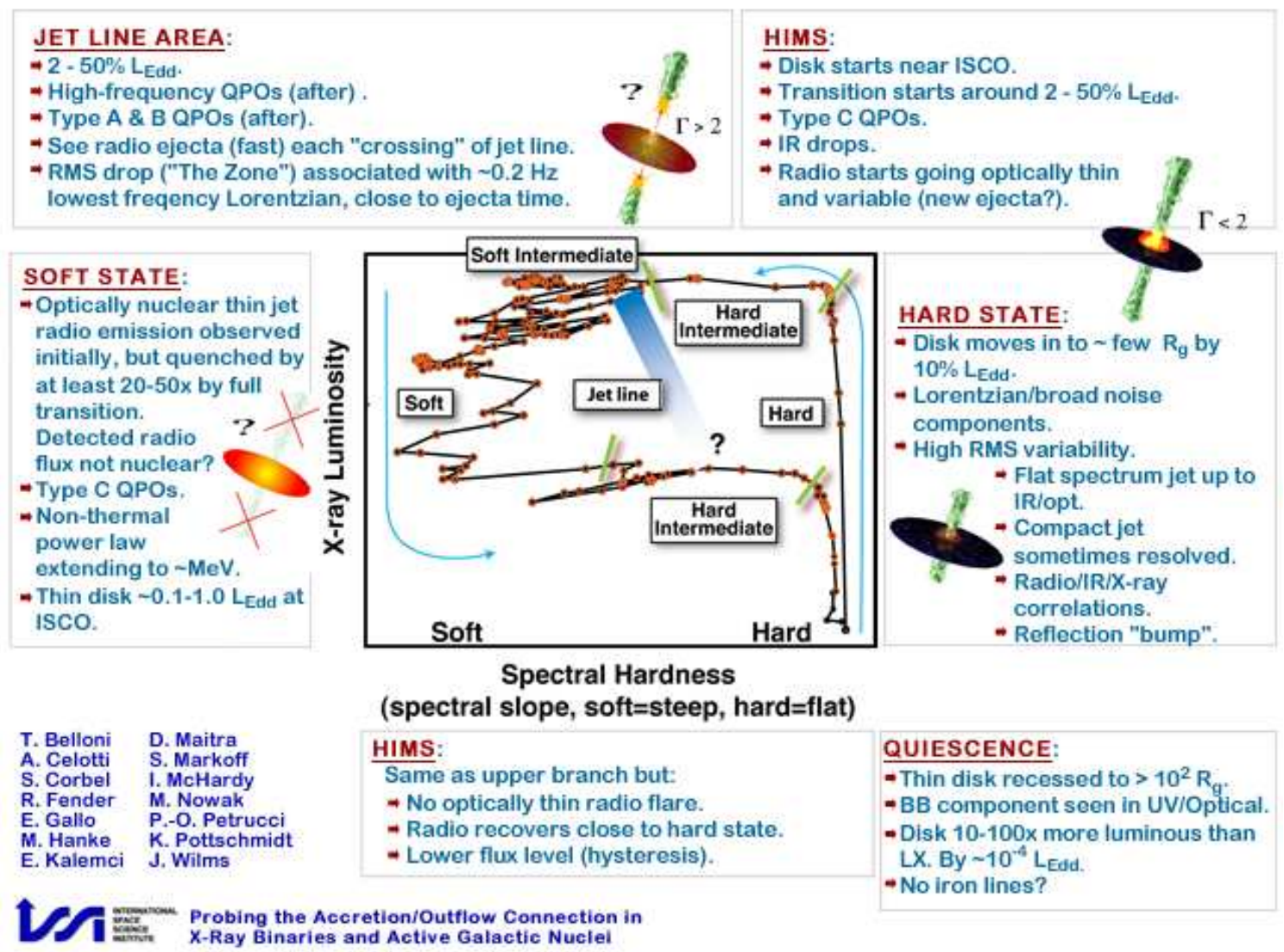

Figure 8: Summary of observational properties of the X-ray and radio emission of black hole X-ray binary transients, and their evolution over an outburst. Here the outburst states are classified by their position in the hardness-intensity diagram, i.e., in the "q" (central panel).

the INTEGRAL epochs. While it does show a brightening/hard and a declining/soft branch as transients do, this HID is rather " $\boldsymbol{p}$-shaped" due to the faint soft state of epoch 01. Following the evolution into and out-off the faint soft state (Fig. 9, red points), a pattern similar to a regular outburst - developing anti-clockwise, from the hard branch over the upper transitional branch, down the soft branch, and through the lower transitional branch back to the hard branch - is observed. Consistent with the conclusion from spectral fitting (see section 1 and [9]) this suggests that the faint soft states correspond to the thermally dominated phase observed during LMXB outburst decays.

We also show the rarely discussed HID for energies above $20 \mathrm{keV}$ (Fig. 10). Since the thermal disk component does not reach into this energy band there is only one hardness branch. Nevertheless the effect of softening in the fainter states is still apparent, indicating that the faint soft state is not entirely due to a change in the relative contribution of the soft and hard spectral components, but that the latter also changes in itself.

Interestingly GRS 1758-258 most likely has a low mass companion, namely a K0 III giant in an $18.45 \pm 0.10$ day binary orbit, and is thus thought to be accreting via Roche lobe overflow $[14,12]$. The accretion process can therefore be expected to show similarities to LMXB transients 


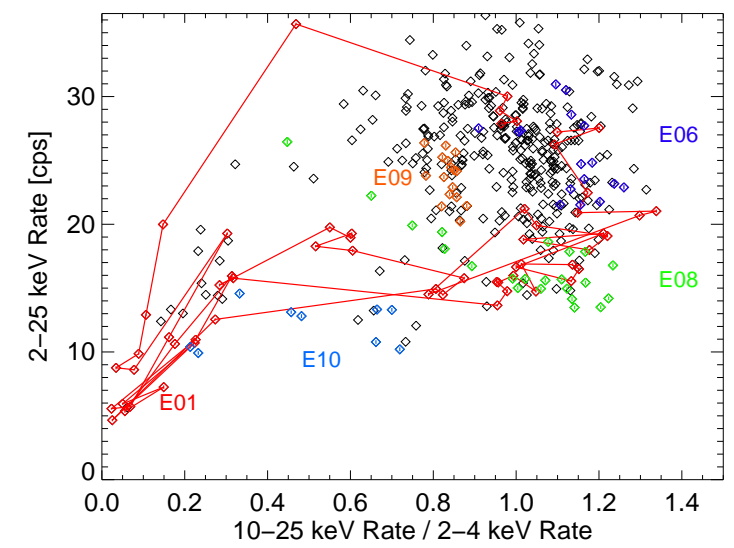

Figure 9: Hardness-intensity diagram for the RXTE-PCA monitoring since 2003 (individual RXTE observations are plotted). Some of the epochs are highlighted in color. The faint soft state of epoch 01 plus several pointings before and after are shown in red to indicate the peculiar $p$-shape of the (anti-clockwise) evolution.

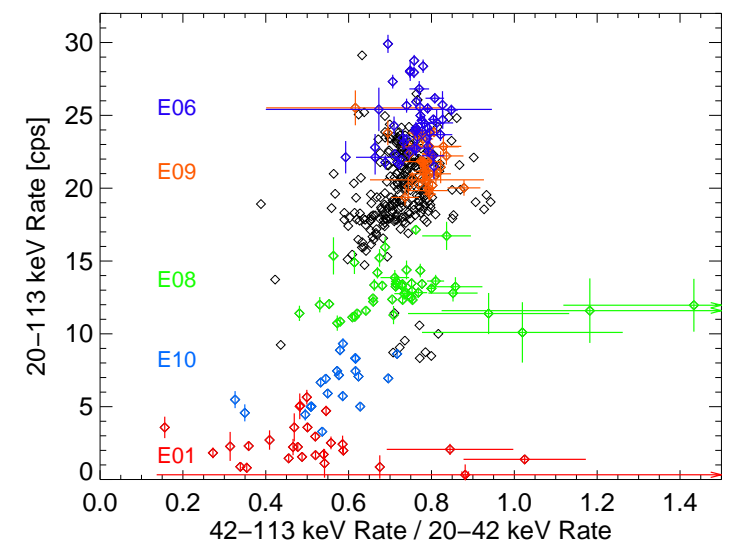

Figure 10: Hardness-intensity diagram for the $I N$ TEGRAL-ISGRI monitoring since 2003 ( $1 \mathrm{~d}$ time bins), i.e., for the hard energy range. Compared to the $<20 \mathrm{keV}$ PCA HID, the $>20 \mathrm{keV}$ ISGRI HID does not show the hysteresis of a soft and a hard branch. Softening, however, is still associated with lower intensities.

and especially to include phases during which the mass transfer is reduced or has stopped completely [15]. In any case GRS 1758-258 represents an important connection between persistent and transient sources ${ }^{3}$ and the faint soft states provide a rare opportunity for studying what happens when the mass transfer is shut off, independent of the possible influence of a preceding outburst.

\section{Conclusions}

\subsection{Summary}

The INTEGRAL and RXTE monitoring campaigns of GRS 1758-258 provide an exceptional database for studying the hard state of black holes. Above we presented the analysis of data from 2003 to 2008, modeling spectra averaged over INTEGRAL viewing epochs as well as a long term average hard state spectrum, and discussing the hardness-intensity diagram above and below $20 \mathrm{keV}$, thereby extending the study down to time scales of days. We report the following main results:

- The epoch averaged 3-300 keV hard state spectra (typical INTEGRAL exposures of 1-2 Ms) can be modeled with a cutoff power law model or thermal Comptonization. They are consistent with interstellar absorption. A weak iron line is detected and sometimes a weak soft excess. This picture is consistent with earlier results.

- On time scales of months $\Gamma$, i.e., the softness of the broad band spectrum, and the 3-300 keV flux are anti-correlated.

\footnotetext{
${ }^{3} 1 \mathrm{E} 1740.7-2942$ might be the only similar case.
} 
- As reported in an earlier analysis of the first four epochs [9], a faint soft state was observed in 2003 spring (epoch 01). Another softer-than-average state was observed in 2007 fall (epoch 10).

- The 2003-2007 average hard state spectrum allows us to detect the source up to $\sim 800 \mathrm{keV}$ with PICsIT. There are indications of a hard tail, i.e., the presence of a non-thermal Comptonization component, above $300 \mathrm{keV}$. In Cyg X-1 such a component has been observed in softer states, i.e., not in the hard state (see, e.g., [1, 3]).

- Although the HID of GRS 1758-258 is rather "p"- instead of "q"-shaped due to the faint soft state, the source can probably be understood in the canonical black hole transient picture, by interpreting the faint soft states as times of reduced mass accretion similar to outburst decays. This is consistent with the K-giant companion candidate, i.e., Roche-lobe overflow accretion.

\subsection{Outlook}

The analysis of this database is work in progress. Plans for future projects include to:

- Refine the broad band spectra as more data and updated calibration becomes available. (INTEGRAL monitoring of GRS $1758-258$ is ongoing and additional data from recent epochs will become public.)

- Investigate whether a model that includes non-thermal Comptonization can describe the long term average hard state spectrum (e.g., eqpair). Include SPI data in this analysis.

- Place further constraints on the soft spectral component in the hard state by (i) evaluating the contribution of INTEGRAL-JEM-X data and (ii) using Swift-XRT data to constrain future epoch averaged spectra (a Swift AO-5 proposal has been submitted). Should INTEGRAL observe another faint soft state we plan to ask for a $S$ wift ToO, in order to follow the evolution of the soft component.

- Compare the $>20 \mathrm{keV}$ HID of GRS 1758-258 and other black hole binaries, as the HID of these sources has generally not been extensively studied at these energies. Model broad band spectra on time scales shorter than the INTEGRAL viewing epochs.

\section{Acknowledgments}

We thank the organizing committee for this great conference in Copenhagen, Denmark, that several of us had the pleasure to attend (KP, DMS, IK, PK)! This work has been partly funded by NASA grant NNX07AG50G (travel KP). This research has also been supported in part by the Polish MNiSW grant NN203065933, and the Polish Astroparticle Network 621/E-78/SN-0068/2007. EK acknowledges support from TÜBITTAK Career Program 106T570, Turkish National Academy of Sciences GEBİP Program, and European Commission FP7 ITN 215212-2 "Black Hole Universe" Project. This work is based on observations with INTEGRAL, an ESA project with instruments and science data centre funded by ESA member states (especially the PI countries: Denmark, France, Germany, Italy, Switzerland, Spain), Czech Republic and Poland, and with the participation 
of Russia and the USA. We thank the RXTE schedulers for making the years long monitoring campaign of GRS $1758-258$ possible.

\section{References}

[1] M. Cadolle Bel, P. Sizun, A. Goldwurm, J. Rodriguez, P. Laurent, A. A. Zdziarski, L. Foschini, P. Goldoni, C. Gouiffès, J. Malzac, E. Jourdain, and J.-P. Roques, The broad-band spectrum of Cygnus X-1 measured by INTEGRAL, A\&A 446, 591-602 (2006).

[2] R. P. Fender, T. M. Belloni, and E. Gallo, Towards a unified model for black hole X-ray binary jets, MNRAS 355, 1105-1118 (2004).

[3] Sonja Fritz, X-ray observations of black hole and neutron star binary systems, Ph.D. thesis, Universität Tübingen, 2008, http: / / tobias-lib.ub.uni-tuebingen.de/volltexte/ $2008 / 3533 / p d f / d i s s \_s f . \% p d f$.

[4] D. Lin, I. A. Smith, E. P. Liang, T. Bridgman, D. M. Smith, J. Martí, P. Durouchoux, I. F. Mirabel, and L. F. Rodríguez, Simultaneous Observations of GRS 1758-258 in 1997 by VLA, IRAM, SEST, RXTE, and OSSE: Spectroscopy and Timing, ApJ 532, 548-562 (2000).

[5] P. Lubiński, Analysis of extremely low signal-to-noise ratio data from INTEGRAL/PICsIT, A\&A (2008), in press [arXiv:0809.0427].

[6] D. S. Main, D. M. Smith, W. A. Heindl, J. Swank, M. Leventhal, I. F. Mirabel, and L. F. Rodríguez, Long-Term X-Ray Monitoring of 1E 1740.7-2942 and GRS 1758-258, ApJ 525, 901-908 (1999).

[7] P. Mandrou, Galactic Center, IAUC 5032 (1990).

[8] S. Markoff, M. A. Nowak, and J. Wilms, Going with the flow: can the base of jets subsume the role of compact accretion disk coronae?, ApJ 635, 1203-1216(2005).

[9] K. Pottschmidt, M. Chernyakova, A. A. Zdziarski, P. Lubiński, D. M. Smith, and N. Bezayiff, INTEGRAL and RXTE monitoring of GRS 1758-258 in 2003 and 2004. A transition from the dim soft state to the hard state, A\&A 452, 285-294 (2006).

[10] R. A. Remillard, X-ray states of black-hole binaries and implications for the mechanism of steady jets, in proceedings of Texas@ Stanford 2004, (P. Chen, ed.), SLAC Electronic Conference Proceedings Archive, 2005, [astro-ph/0504129].

[11] L. F. Rodriguez, I. F. Mirabel, and J. Marti, The radio counterpart of the hard X-ray source GRS 1758-258, ApJ Lett. 401, L15-L18 (1992).

[12] D. M. Rothstein, S. S. Eikenberry, S. Chatterjee, E. Egami, S. G. Djorgovski, and W. A. Heindl, The Infrared Counterpart of the Microquasar GRS 1758-258, ApJ Lett. 580, L61-L63 (2002).

[13] D. M. Smith, W. A. Heindl, C. B. Markwardt, and J. H. Swank, A Transition to the Soft State in GRS 1758-258, ApJ Lett. 554, L41-L44 (2001).

[14] D. M. Smith, W. A. Heindl, and J. H. Swank, Orbital and Superorbital Periods of 1E 1740.7-2942 and GRS 1758-258, ApJ Lett. 578, L129-L132 (2002).

[15] D. M. Smith, W. A. Heindl, and J. H. Swank, Two Different Long-Term Behaviors in Black Hole Candidates: Evidence for Two Accretion Flows, ApJ 569, 362-380 (2002).

[16] D. M. Smith, W. A. Heindl, J. H. Swank, and C. B. Markwardt, Black-Hole Candidate GRS 1758-258 Enters an "Off" State, ATEL 66 (2001). 
[17] R. Sunyaev, E. Churazov, M. Gilfanov, M. Pavlinsky, S. Grebenev, G. Babalyan, I. Dekhanov, N. Yamburenko, L. Bouchet, M. Niel, J. P. Roques, P. Mandrou, A. Goldwurm, B. Cordier, P. Laurent, and J. Paul, Two hard X-ray sources in 100 square degrees around the Galactic Center, A\&A 247, L29-L32 (1991).

[18] J. Wilms, K. Pottschmidt, G. G. Pooley, S. Markoff, M. A. Nowak, I. Kreykenbohm, and R. E. Rothschild, Correlated Radio-X-Ray Variability of Galactic Black Holes: A Radio-X-Ray Flare in Cygnus X-1, ApJ Lett. 663, 97-100 (2007).

[19] A. A. Zdziarski, M. Gierliński, J. Mikołajewska, G. Wardziński, D. M. Smith, B. Alan Harmon, and S. Kitamoto, GX 339-4: the distance, state transitions, hysteresis and spectral correlations, MNRAS 351, 791-807 (2004).

[20] A. A. Zdziarski and M. Gierliński, Radiative Processes, Spectral States and Variability of Black-Hole Binaries, Progress of Theoretical Physics Supplement 155, 99-119 (2004). 